\title{
Label-free quantitative proteomic analysis identifies CTNNB1 as a direct target of FOXP3 in gastric cancer cells
}

\author{
DU-YI PAN ${ }^{1 *}$, XIAO-QING ZENG ${ }^{1 *}$, GUI-FEN MA $^{1}$, JING GAO $^{2}$, NA LI $^{1}$, QING MIAO ${ }^{1}$, \\ JING-JING LIAN $^{1}$, HU ZHOU ${ }^{2}$, LI-LI XU ${ }^{1}$ and SHI-YAO CHEN ${ }^{1}$ \\ ${ }^{1}$ Department of Gastroenterology, Zhongshan Hospital, Fudan University, Shanghai 200032; \\ ${ }^{2}$ Shanghai Institute of Materia Medica, Chinese Academy of Sciences, Shanghai 201203, P.R. China
}

Received June 2, 2016; Accepted February 15, 2018

DOI: $10.3892 / 01.2018 .8277$

\begin{abstract}
Forkhead box protein 3 (FOXP3) is expressed in numerous types of tumor cell and is associated with tumor progression and prognosis. A previous study reported that FOXP3 inhibited cellular proliferation and induced apoptosis of gastric cancer (GC) cells by activating the apoptosis signaling pathway. In the present study, label-free quantitative proteomic analysis and chromatin immunoprecipitation-polymerase chain reaction (ChIP-PCR) was performed to investigate the mechanism by which the anticancer role ofFOXP 3 was mediated and the proteins that with which it may interact. Label-free quantitative proteomic analysis was used to screen for proteins differentially expressed between FOXP3-overexpressing GC (AF) and vector (ANC) cells. Catenin $\beta 1$ (CTNNB1) was one of the proteins that exhibited the greatest difference between AF and ANC among 3,313 proteins identified by liquid chromatography with tandem mass spectrometry analysis. The expression of CTNNB1 was evaluated by reverse transcription-quantitative PCR and western blotting. The association between FOXP3 and CTNNB1 was confirmed by ChIP-PCR in AGS cells. The changes in expression of epithelial-mesenchymal transition-associated proteins were analyzed by western blotting. The level of FOXP3 expression was positively associated with CTNNB1 and E-cadherin expression, but not with vimentin and $\mathrm{N}$-cadherin expression. FOXP3 positively regulates CTNNB1 and binds to it directly. Along with the upregulation of glycogen synthase kinase $3 \beta$ (GSK3 $\beta$ ), which was also a protein whose expression was found to change significantly in proteomic analysis and has a
\end{abstract}

Correspondence to: Professor Shi-Yao Chen, Department of Gastroenterology, Zhongshan Hospital, Fudan University, 180 Fenglin Road, Shanghai 200032, P.R. China

E-mail: chen.shiyao@zs-hospital.sh.cn

*Contributed equally

Key words: forkhead box protein 3, catenin $\beta 1$, label-free quantitative proteomic analysis, gastric cancer, chromatin immunoprecipitation-polymerase chain reaction key role in the Wnt pathway. This association is an attractive and novel hypothesis for the mechanism by which FOXP3 inhibits the invasion and metastasis of GC cells.

\section{Introduction}

Gastric cancer (GC) is a malignant tumor of the gastrointestinal tract that is common worldwide (1). Although the incidence of GC has decreased since the 1990s in the majority of developed countries, outcomes in patients with advanced GC remain poor, and the 5-year survival rate ranges between 4 and $20 \%$ for patients with surgically resected tumors (2). Invasion and metastasis of GC are the two main reasons for its poor prognosis. Forkhead box protein 3 (FOXP3) is a transcription factor that is necessary for the induction of the immunosuppressive functions in regulatory $\mathrm{T}$ cells (3). Previous studies reported the expression of FOXP3 has been observed in a number of types of tumor cell $(4,5)$. The expression of FOXP3 in different tumor cells may drive different functions. In a previous study, high FOXP3 expression in GC cells was found to predict longer survival times (6). FOXP3 was also observed to inhibit proliferation and induce apoptosis in GC cells by activating the apoptotic signaling pathway (7). FOXP3 is reported to negatively regulate nuclear factor $\kappa$-light-chain-enhancer of activated B cells (NF- $\kappa B$ ) and thus has a tumor suppressor role in GC (8). However, the mechanisms by which FOXP3 acts as a tumor suppressor remain largely unknown.

$\mathrm{GC}$ is a polygenic disease linked with the transcription and expression of multiple oncogenes and tumor suppressor genes, which finally express in the form of protein. Label-free quantitative proteomic analysis is a newly emerged, efficient, powerful and cost-effective approach for comparing various proteins from different samples (9). Label-free proteomic analysis can screen differential protein expression with a high efficiency (10). In the present study, cells were digested by the filter-aided sample preparation (FASP) procedure, to obtain purer peptides and higher image quality. FOXP3-overexpressing AGS cells were used as the experimental group and empty vector-transfected AGS cells as the control group. A total of 3,313 protein groups were quantified under highly rigorous criteria with a false discovery rate of $<1 \%$ for peptide and protein groups. Of these proteins, 
276 exhibited differences in expression that were statistically significant between FOXP3-overexpressing and control cells.

In the present study, label-free proteomic analysis was performed to investigate the molecules through which FOXP3 mediates its anticancer role and to obtain a better understanding of its mechanism of action in GC.

\section{Materials and methods}

Cell lines, reagents. Human GC cells AGS were purchased from the Chinese Academy of Science (Shanghai, China). The AGS cells were cultivated in RPMI 1640 medium (Hyclone; GE Healthcare Life Sciences, Logan, UT, USA) containing $10 \%$ fetal bovine serum (Invitrogen; Thermo Fisher Scientific, Inc., Waltham, MA, USA) at $37^{\circ} \mathrm{C}$ in $5 \% \mathrm{CO}_{2}$.

Transfection. The lentivirus of FOXP3 overexpression and its vehicle were purchased from Genepharma (Shanghai GenePharma Co., Ltd., Shanghai, China). The AGS cells were grown to $70-80 \%$ confluence in six well plates. The AGS cells were infected with a multiplicity of infection (MOI) of 50 to generate FOXP3-overexpression (referred to as AF). Cells infected with an empty vector served as control cells (referred to as ANC). A total of $2.5 \mu \mathrm{g} / \mathrm{ml}$ puromycin was added to AF cells $48 \mathrm{~h}$ after transfection to select cells stably expressing FOXP3. Subsequent experiments were conducted immediately following the selection.

Protein preparation and label-free proteomic analysis. Cells were digested using the FASP procedure, as described previously (11). Briefly, $10^{7}$ AGS cells were washed with phosphate buffered saline (PBS) three times to remove, then lysed using SDT buffer (containing 4\% SDS (m/w), $100 \mathrm{mM}$ DTT and $100 \mathrm{mM}$ Tris, $\mathrm{pH}$ 7.6). The lysate was incubated at $95^{\circ} \mathrm{C}$ for $5 \mathrm{~min}$ and then centrifuged at $15,000 \mathrm{x} \mathrm{g}$ for $10 \mathrm{~min}$ at $4^{\circ} \mathrm{C}$, and the supernatant was used for proteomics sample preparation. Label-free proteomic analysis was performed as previously described (9). The reverse phase high-performance liquid chromatography (HPLC) separation was achieved using the EASY-nLC1000 HPLC system (Thermo Fisher Scientific, Inc.) using a self-packed column (75 $\mu \mathrm{m}$ x150 mm; $3 \mu \mathrm{m}$ ReproSil-Pur C18 beads, $120 \mathrm{~A}^{\circ}$; Dr. Maisch HPLC $\mathrm{GmbH}$, Ammerbuch, Germany) at a flow rate of $300 \mathrm{nl} / \mathrm{min}$ using 240 min gradients. The full mass was scanned in the Orbitrap analyzer with $\mathrm{R}=70,000$ (defined at $\mathrm{m} / \mathrm{z} 200$ ), and the subsequent $\mathrm{MS} / \mathrm{MS}$ analyses were performed with $\mathrm{R}=17,500$. Proteins were identified by searching the MS and MS/MS data for the peptides against a decoy version of the International Protein Index human or rat database (version 3.87, 91464 protein sequences; European Bioinformatics Institute, Hinxton, Cambridge, UK). Trypsin/P was selected as the digestive enzyme with two potential missed cleavages. Protein abundance was calculated according to the normalized spectral protein intensity (LFQ intensity).

Reverse transcription-quantitative polymerase chain reaction (RT-qPCR). Total RNA was isolated using commercial RNA isolation kit (Takara Biotechnology Co., Ltd., Dalian, China) according to the manufacturer's protocol, and $2 \mu \mathrm{g}$ RNA was reverse transcribed to cDNA using oligodT primers with the
Primer Script ${ }^{\mathrm{TM}}$ RT Reagent (Takara Biotechnology Co., Ltd.). The target genes FOXP3, catenin $\beta 1$ (CTNNB1), E-cadherin, $\mathrm{N}$-cadherin, and vimentin, and the internal control gene GAPDH were then amplified by qPCR. The RT-qPCR was performed in a total volume of $10 \mu \mathrm{l}$, with $2 \mathrm{X} \mathrm{SYBR}{ }^{\circledR}$ Green Mix (Takara Biotechnology Co., Ltd.) in the ABI PRISM 7500 system (PerkinElmer, Inc., Waltham, MA, USA). All samples were run in triplicate. The amplification reaction was initiated by denaturing DNA at $95^{\circ} \mathrm{C}$ for $5 \mathrm{~min}$, followed by 40 cycles of template denaturing at $94^{\circ} \mathrm{C}$ for $1 \mathrm{~min}$, primer annealing at $60^{\circ} \mathrm{C}$ for $1 \mathrm{~min}$, and primer extension at $72^{\circ} \mathrm{C}$ for $1 \mathrm{~min}$. The comparative $\mathrm{Cq}$ method $\left(2^{-\Delta \Delta \mathrm{Cq}}\right.$ method) was used for RT-qPCR data analysis (12).

The primers used were as follows: FOXP3 forward, 5'-AAG CAGCACTACATTGACCTGAAA-3' and reverse, 5'-GGT CTCCCCAAGCATCACTC-3'; CTNNB1 forward, 5'-TGG TGACAGGGAAGACATCA-3' and reverse, 5'-CCATAGTGA AGGCGAACTGC-3'; E-cadherin forward, 5'-GGTCTCTCT CACCACCTCCA-3' and reverse, 5'-CCTCGGACACTTCCA CTCTC-3'; N-cadherin forward, 5'-CGTGAAGGTTTGCCA GTGT-3' and reverse, 5'-CAGCACAAGGATAAGCAGGA-3'; vimentin forward, 5'-GTACCGGAGACAGGTGCAGT-3' and reverse, 5'-AACGGCAAAGTTCTCTTCCA-3'; and GAPDH forward, 5'-GCACCGTCAAGGCTGAGAAC-3' and reverse, 5'-TGGTGAAGACGCCAGTGGA-3'.

Western blot analysis. Cells were collected and proteins were extracted with radioimmunoprecipitation assay lysis buffer (Nanjing KeyGen Biotech Co., Ltd., Nanjing, China). The protein concentration was determined by the BCA protein assay (Shanghai Yeasen Biotech Co., Ltd., Shanghai, China). Protein samples (40 $\mu \mathrm{g}$ per lane) were separated by $10 \%$ SDS-PAGE and then electro-transferred onto nitrocellulose membranes for $90 \mathrm{~min}$. The membranes were blocked for $1 \mathrm{~h}$ with $5 \%$ skimmed milk at room temperature and incubated with primary antibodies overnight at $4^{\circ} \mathrm{C}$, followed by incubation with secondary antibodies. Primary antibodies used were as follows: Specific to FOXP3 (cat no. 236A/E7; 1:500; anti-mouse; Abcam, Cambridge, MA, USA), CTNNB1 (cat no. 8480; 1:1,000; anti-rabbit), E-cadherin (cat no. 3195; 1:1,000; anti-rabbit), $\mathrm{N}$-cadherin (cat no. 13116; 1:1,000; anti-rabbit), Vimentin (cat no. 5741; 1:1,000; anti-rabbit), GSK3 $\beta$ (cat no. 12456; 1:1,000; anti-rabbit) and GAPDH (cat no. 2118; 1:5,000; anti-mouse) (all purchased from Cell Signaling Technology, Inc., Danvers, MA, USA). Secondary antibodies used were horseradish peroxidase-conjugated anti-rabbit/mouse immunoglobulin G (cat no. 111-035-003/115-035-003; 1:5,000; Jackson Laboratory, Bar Harbor, ME, USA). The reactions were visualized using enhanced chemiluminescence kit (Merck KGaA, Darmstadt, Germany) and a gel imaging system.

Chromatin immunoprecipitation followed by PCR (ChIP-PCR). ChIP was performed in native conditions. In brief, cells at a concentration of $2 \times 10^{6} / \mathrm{ml}$ were treated with $1 \%$ formaldehyde for $10 \mathrm{~min}$ at room temperature. Glycine $(1.25 \mathrm{M})$ was then added and the samples were incubated for $5 \mathrm{~min}$ at room temperature. The cells were washed twice with cold PBS and pelleted. Each pellet was resuspended in $1 \mathrm{ml}$ of lysis buffer (50 mM Tris, pH 7.4, 150 mM NaCl, 1 mM EDTA, 0.1\% SDS, $1 \%$ NP-40, $0.5 \%$ sodium deoxycholate, $0.5 \mathrm{mM}$ DTT and $1 \mathrm{mM}$ 
A

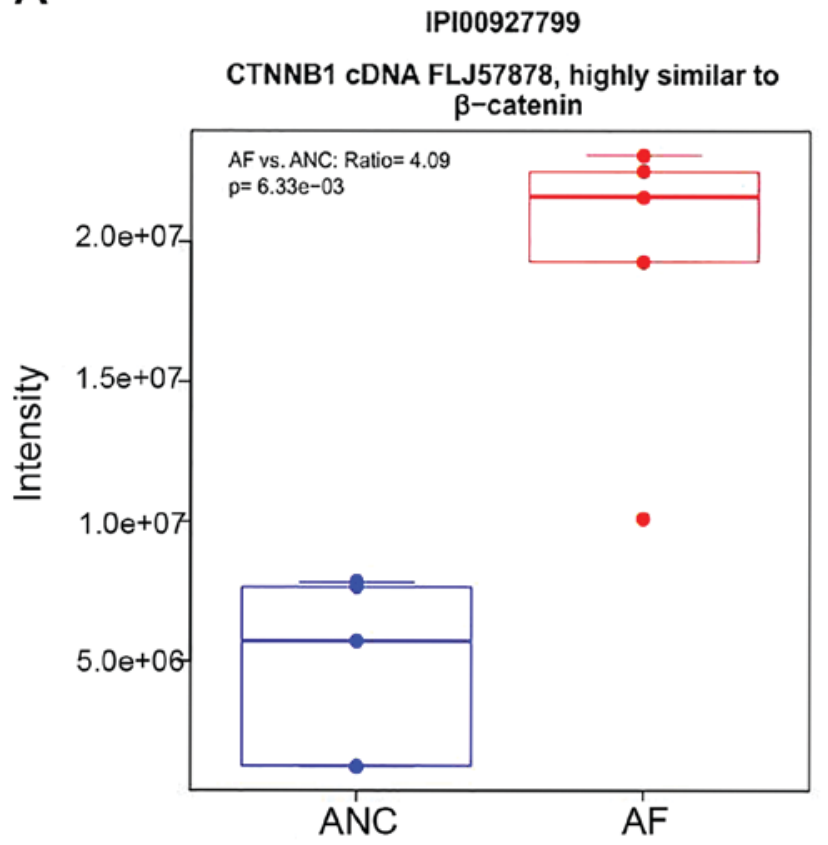

B

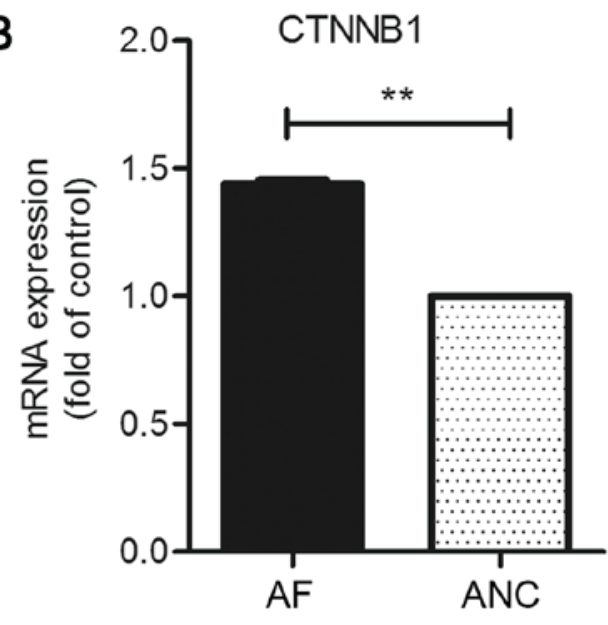

C

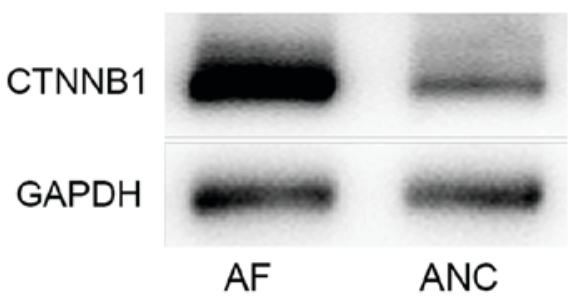

Figure 1.FOXP3 is associated with the expression of CTNNB1. (A) Proteomic analysis showing box and whisker plots for the label-free quantification intensity of CTNNB1 ( $\mathrm{P}=0.006$; fold change, 4.09). (B) RT-qPCR of the mRNA expression level of CTNNB1. (C) Western blot analysis of CTNNB1. * P<0.01. FOXP3, forkhead box protein 3; CTNNB1, catenin $\beta 1$; RT-qPCR, reverse transcription-quantitative polymerase chain reaction.

phenylmethylsulfonyl fluoride/cocktail), incubated on ice with vortexing for $10 \mathrm{~min}$, and the lysate was obtained by centrifugation at $4^{\circ} \mathrm{C}$ with $13,000 \mathrm{x}$ for $10 \mathrm{~min}$. The majority of the DNA fragments were sheared by sonication on ice to a length of 200-500 bp. Antibodies specific to FOXP3 (cat no. ab2481, 1:50, Abcam), RNA pol2 (cat no. sc-899, 1:100, Santa Cruz Biotechnology, Inc., Dallas, TX, USA) and protein G beads were added and samples were incubated for $4 \mathrm{~h}$ at $4^{\circ} \mathrm{C}$. The samples were washed once in a low-salt wash buffer $(20 \mathrm{mM}$ Tris- $\mathrm{HCl}$, pH $8.0150 \mathrm{mM} \mathrm{NaCl}, 0.1 \%$ SDS, $1 \%$ Triton $\mathrm{X}-100$, $2 \mathrm{mM}$ EDTA), once in a high-salt wash buffer (20 mM Tris- $\mathrm{HCl}$, pH 8.0, $500 \mathrm{mM} \mathrm{NaCl}, 0.1 \%$ SDS, $1 \%$ Triton X-100, $2 \mathrm{mM}$ EDTA), once in a $\mathrm{LiCl}$ wash buffer (0.25 M LiCl, $1 \% \mathrm{NP}-40,1 \%$ sodium deoxycholate, $1 \mathrm{mM}$ EDTA, $10 \mathrm{mM}$ Tris-HCl, $\mathrm{pH}$ 8.0) and once in a TE buffer $(10 \mathrm{mM}$ Tris- $\mathrm{HCl}, \mathrm{pH} 8.0,1 \mathrm{mM}$ EDTA). The beads were then resuspended in lysis buffer and treated with proteinase $\mathrm{K}$ at $45^{\circ} \mathrm{C}$ for $45 \mathrm{~min}$. Co-precipitated DNAs were purified with phenol-chloroform to eliminate SDS sediment. The effectiveness of CHIP was detected using PCR. The PCR was performed to verify whether these DNA fragments were between 200-500 bp using the 2X Hieff ${ }^{\text {тм }}$ HotStart PCR Master Mix (Yeasen Biotechnology Co., Ltd., Shanghai, China) with DNA ladder (cat no. D0107, Beyotime Institute of Biotechnology, Haimen, China) as the marker. The sequences of primers were: FOXP3 forward, 5'-AAGCAGCACTACATT GACCTGAAA-3'; FOXP3 reverse, 5'-GGTCTCCCCAAG CATCACTC-3'; GAPDH forward, 5'-ATGGGGCGCACG GCGGGAATG-3'; GAPDH reverse 5'-CTCCTTGGGCGC TTCGGCC-3'. The thermocycling conditions were 35 cycles of $95^{\circ} \mathrm{C}$ for $30 \mathrm{sec}$ for denaturation, $55^{\circ} \mathrm{C}$ for $30 \mathrm{sec}$ for annealing and $72^{\circ} \mathrm{C}$ for $30 \mathrm{sec}$ for elongation. PCR products were loaded onto a $1 \%$ agarose gel, and the bands were visualized using the
Molecular Imager Gel Doc XR system (Bio-Rad Laboratories, Inc., Hercules, CA, USA).

Statistical analysis. All data were analyzed using the SPSS 20.0 statistical program (IBM Corp., Armonk, NY, USA). Values are expressed as fold change or mean \pm standard error of the mean. Unpaired Student's t-tests were used for comparisons between two means. $\mathrm{P}<0.05$ was considered to indicate statistical significance.

\section{Results}

Label-free quantitative proteomic analysis of $A F$ and $A N C$ cells. In an attempt to identify changes in proteins, the differential protein expression between the AF and the ANC cells was monitored. To ensure the reproducibility of the results, five biological replicates were performed for proteomic analysis. The high homogeneity of AF and ANC resulted in highly accurate liquid chromatography with tandem mass spectroscopy identification results. Of a total of 3,313 proteins, the expression of 276 differed significantly $\left(\mathrm{P}<0.05\right.$, using a $\log _{2}$ fold-change $>2$ as the cut-off). The expression of CTNNB1 in AF cells was 4.09-fold higher than that in the control cells (Fig. 1A). The level of mRNA expression of CTNNB1 in AF and ANC was verified by RT-qPCR (Fig. 1B). The level of expression of the protein was determined by western blot analysis (Fig. 1C).

Expression of epithelial-mesenchymal transition (EMT)-associated proteins does not differ significantly between AF and ANC. The results of RT-qPCR and western blot analysis indicated that levels of the epithelial phenotype 
A

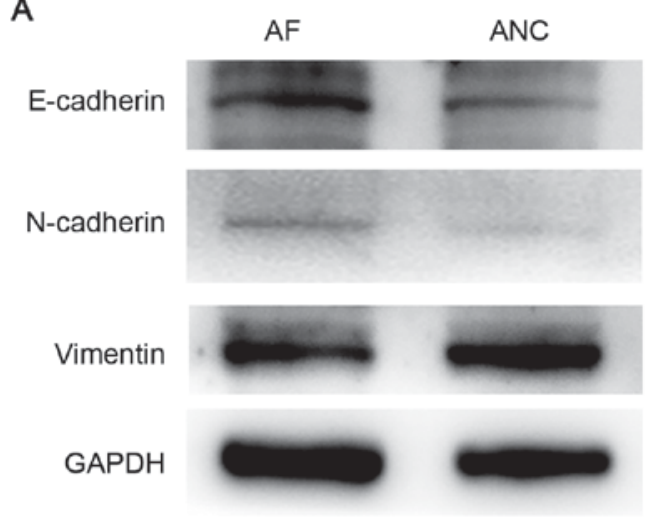

C

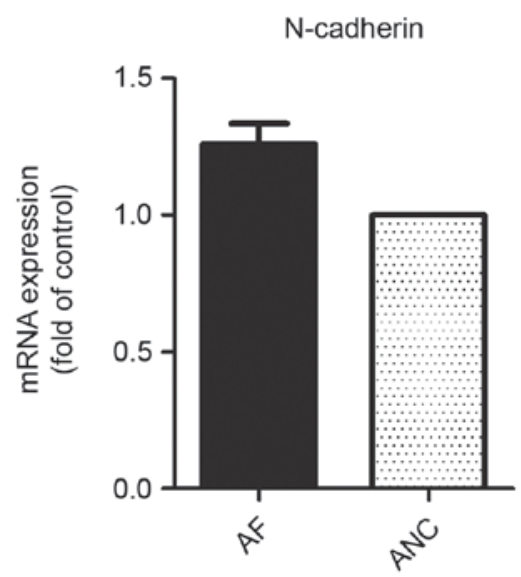

B
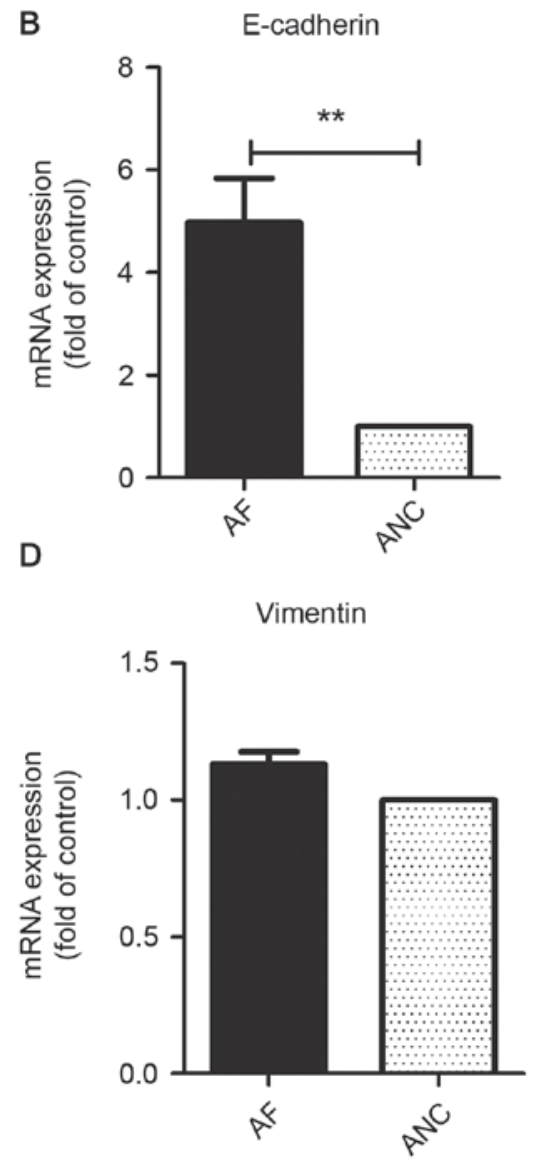

Figure 2. Expression levels of E-cadherin, $\mathrm{N}$-cadherin and vimentin. (A) E-cadherin expression was upregulated in AF cells, but that of vimentin or $\mathrm{N}$-cadherin were not significantly different. (B-D) The results of reverse transcription-quantitative polymerase chain reaction for (B) E-cadherin, (C) N-cadherin and (D) vimentin match those of the western blot. ${ }^{* *} \mathrm{P}<0.01$. AF, cells overexpressing FOXP3; ANC, cells transfected with an empty vector.

protein E-cadherin and CTNNB1 were significantly elevated in $\mathrm{AF}$, but those of the mesenchymal phenotype proteins vimentin and $\mathrm{N}$-cadherin were not (Fig. 2).

Glycogen synthase kinase 3- $\beta$ (GSK3 $\beta)$ is upregulated in AF cells. GSK $3 \beta$ is a key member of the destruction complex that can degrade the CTNNB1 in cytoplasm rapidly (13). Cytoplasmic levels of CTNNB1 are tightly controlled by GSK $3 \beta$ and the degradation of CTNNB1 in cytoplasm could inhibit the Wnt pathway (13). The expression of GSK3 $\beta$ in AF cells was found to be 1.49-fold higher than in the control cells. (Fig. 3A) The level of the protein was determined by western blot analysis (Fig. 3B).

FOXP3 interacts with CTNNB1 in AGS cells. The observation that the protein level of CTNNB1 was affected by FOXP3 has been supported by label-free proteomic analysis, and indicates that FOXP3 may modulate the former to a certain degree. To investigate whether FOXP3 affected CTNNB1 by binding to it, a ChIP-PCR assay was performed. ChIP-PCR assays revealed that FOXP3 could bind directly to the promoter region of CTNNB1 in AGS cells (Fig. 4). There were three regions to which FOXP3 could bind. The specific FOXP3 binding positions were located between $-1,502$ and $-1,251 \mathrm{bp},-1,002$ and $-751 \mathrm{bp}$, and -751 and $500 \mathrm{bp}$, starting from the transcription site of the CTNNB1 gene promoter. Data from ChIP-PCR indicated that FOXP3 could control CTNNB1 directly, and thus affect the downstream pathways.

\section{Discussion}

In summary, the results of the present study revealed a novel function of FOXP3, acting as a tumor suppressor through interaction with CTNNB1. In a previous study, FOXP3-positive staining was found to associate with a favorable prognosis in patients with GC, and that upregulation of the FOXP3 gene inhibits GC cell growth in vitro and in vivo (6). Several mechanisms, including the activation of the apoptotic signaling pathway and inhibition of NF- $\mathrm{NB}$ activity, have been implicated in the anticancer activity of FOXP3 in GC $(7,8)$.

Label-free quantitative proteomic analysis identified CTNNB1 as a novel FOXP3-interacting partner. Consistent with former results, the epithelial phenotype E-cadherin and CTNNB1 were upregulated in FOXP3-overexpressing AGS cells. EMT occurs due to the induction of transcription factors, which alter gene expression to promote the loss of cell-cell adhesion, leading to a shift in cytoskeletal dynamics and a change from epithelial morphology and physiology to the mesenchymal phenotype (14). Cells lose their epithelial characteristics, instead gaining an invasive and migratory mesenchymal phenotype, allowing these cells to leave the tissue parenchyma and enter the systemic 
A

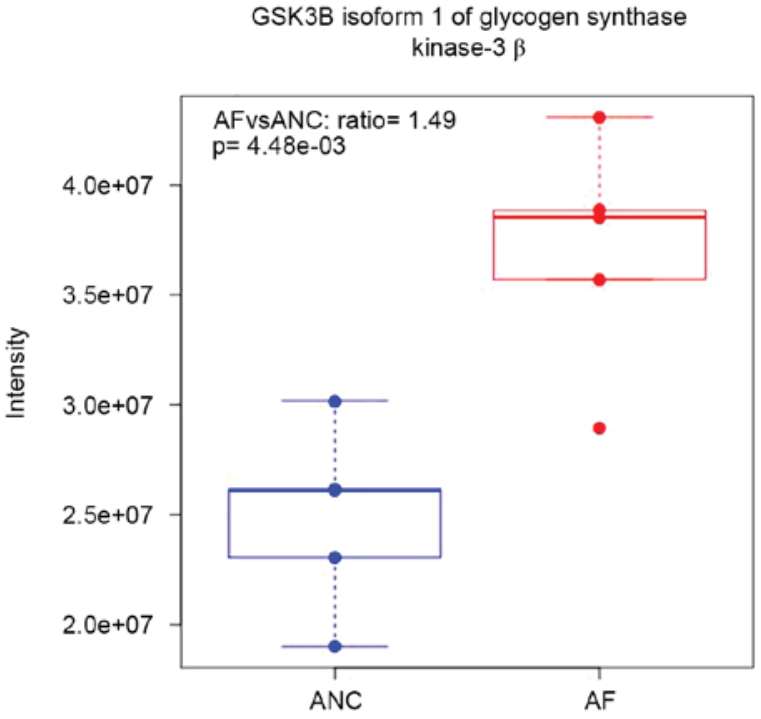

B

IPI00028570

1 of glycogen synthase

$\mathrm{AF}$

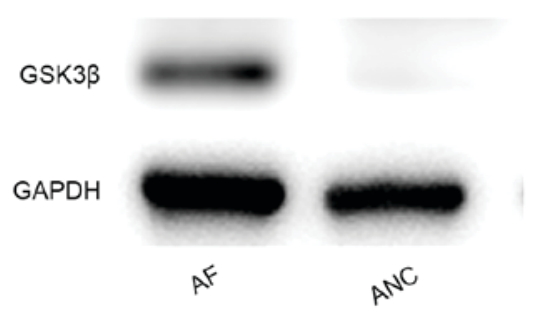

Figure 3. GSK $3 \beta$ levels in ANC and AF cells. (A) Proteomic analysis showing the label-free quantification intensity of GSK $3 \beta(P=0.004$; fold change, 1.49). (B) Western blot analysis confirmed the upregulation of GSK $3 \beta$. GSK3 $\beta$, glycogen synthase kinase $3 \beta ; A F$, cells overexpressing forkhead box protein 3; ANC, cells transfected with an empty vector.

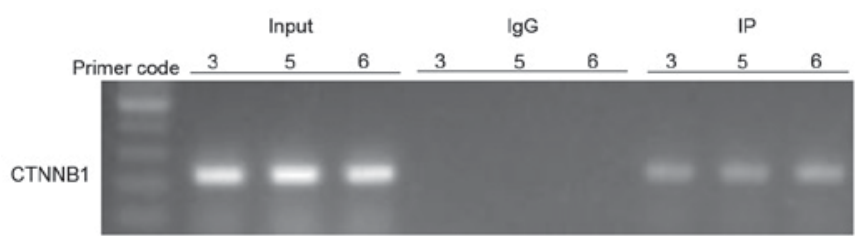

Figure 4. Chromatin immunoprecipitation-polymerase chain reaction verification of FOXP3 binding sites. FOXP3 specifically bound to positions located between $-1,502$ and $-1,251$ bp (primer no. 3); -1,002 and -751 bp (primer no. 5); -751 and 500 bp (primer no. 6) from the start site of the CTNNB1 gene promoter. Input, antigen protein used as positive control; IgG immunoglobulin G negative control; IP, product of immunoprecipitation; FOXP3, forkhead box protein 3; CTNNB1, catenin $\beta 1$.

circulation during cancer metastasis. One hallmark of EMT is the dissociation of the E-cadherin-CTNNB1- $\alpha$-catenin complex from the membrane (15). Thus, the loss of expression of E-cadherin is always accompanied by the loss of expression of CTNNB1 at the membrane. The mesenchymal phenotype indicators vimentin and $\mathrm{N}$-cadherin are always upregulated in EMT.

Given that the role of FOXP3 in EMT has not been investigated previously, the present study measured the mRNA and protein levels of mesenchymal phenotype indicators vimentin and $\mathrm{N}$-cadherin to determine whether
FOXP3 could inhibit EMT. However, the results observed were not expected. Vimentin and $\mathrm{N}$-cadherin were not downregulated in FOXP3-overexpressing cells, indicating that FOXP3 does not mediate its anticancer effect through a mesenchymal-to-epithelial transition. However, using ChIP-PCR, it was identified that FOXP3 could bind directly to the promoter region of CTNNB1. There are three binding domains, located between $-1,502$ and $-1,251 \mathrm{bp},-1,002$ and $-751 \mathrm{bp}$, and -751 and $-500 \mathrm{bp}$. These results indicate that FOXP3 upregulates the expression of CTNNB1 in AGS cells.

CTNNB1, commonly known as $\beta$-catenin, was first identified as an adhesion molecule by Imhof et al (16) in the 1980s. Interest in this molecule increased when it was identified that CTNNB1 is important in the initiation and metastasis of cancer (17). CTNNB1 is a dual-function protein: When there is no Wnt signaling, CTNNB1 dynamically links E-cadherin and $\alpha$-catenin at the plasma membrane (18). Adhesion to the basement membrane and to adjacent cells is critical for maintaining the epithelial phenotype (19). In the absence of Wnt signaling, CTNNB1 is rapidly degraded by a destruction complex consisting of adenoma polyposis coli, axin, casein kinase and GSK3 $\beta$ (13). Thus, the concentration of CTNNB1 is maintained at the appropriate level in the cytoplasm. By contrast, in the Wnt signaling pathway the GSK $3 \beta$-dependent phosphorylation of CTNNB1 is inhibited; this results in an accumulation of CTNNB1 in the nucleus, where it functions as a transcriptional activator in conjunction with lymphoid enhancer factor/T-cell factor DNA binding proteins and induces EMT $(20,21)$. The signaling function of CTNNB1 is regulated principally through the alteration of its stability in the cytoplasm.

The present label-free quantitative proteomic analysis also identified GSK3 $\beta$ as a significantly altered protein in AF cells. The expression of GSK $3 \beta$ was 1.49 -fold higher in AF than in APC cells. Since GSK3 $\beta$ could catalyze the phosphorylation of serine or threonine residues on CTNNB1 substrates, the upregulation of GSK $3 \beta$ may promote the degradation of CTNNB1 in the cytoplasm and inhibit the Wnt pathway (18), which, together with the increase in CTNNB1 expression on the membrane, could strengthen the E-cadherin-CTNNB1 complex, making it difficult for the GC cells to move.

In summary, proteomic analysis revealed that CTNNB1 levels were significantly upregulated in FOXP3-overexpressing AGS cells. ChIP-PCR revealed that FOXP3 could bind directly to the promoter region of CTNNB1, which means that FOXP3 could directly regulate CTNNB1. These results provide novel information concerning the anticancer mechanism of FOXP3. Further research is required to investigate the possible pathways in which CTNNB1 is involved with FOXP3.

\section{Acknowledgements}

The authors would like to thank the Key Laboratory of Zhongshan Hospital of Fudan University (Shanghai, China) for the support of cellular experiments, and the Institutional Technology Service Center of Shanghai Institute of Materia Medica (Shanghai, China) for the support of the proteomic experiments. 


\section{Funding}

The present study was supported by the Natural Science Foundation of Shanghai (no. 14ZR1406600) and the Natural Science Foundation for Young Scholars of China (no. 81502005).

\section{Availability of data and materials}

The datasets used and/or analyzed during the current study are available from the corresponding author on reasonable request.

\section{Authors' contributions}

All authors contributed to the conception, design and completion of the present study. DYP and XQZ contributed equally to study design, experimental work and manuscript preparation. GFM designed the study, JG and HZ performed the proteomic experiments, QM and NL analyzed the data and LLX and SYC contributed to refining the study design.

\section{Ethics approval and consent to publish}

No human participants, human data or human tissue were involved in the present study.

\section{Consent for publication}

Not applicable.

\section{Competing interests}

The authors declare that they have no competing interests.

\section{References}

1. Siegel RL, Miller KD and Jemal A: Cancer statistics, 2015. CA Cancer J Clin 65: 5-29, 2015.

2. Washington $\mathrm{K}: 7$ th edition of the AJCC cancer staging manual: Stomach. Ann Surg Oncol 17: 3077-3079, 2010.

3. Hori S and Sakaguchi S: Foxp3: A critical regulator of the development and function of regulatory T cells. Microbes Infect 6: 745-751, 2004.

4. Karanikas V, Speletas M, Zamanakou M, Kalala F, Loules G, Kerenidi T, Barda AK, Gourgoulianis KI and Germenis AE: Foxp3 expression in human cancer cells. J Transl Med 6: 19, 2008.

5. Matsuura K, Yamaguchi Y, Osaki A, Ohara M, Okita R, Emi A, Murakami S and Arihiro K: FOXP3 expression of micrometastasis-positive sentinel nodes in breast cancer patients. Oncol Rep 22: 1181-1187, 2009.
6. Ma GF, Miao Q, Liu YM, Gao H, Lian JJ, Wang YN, Zeng XQ, Luo TC, Ma LL, Shen ZB, et al: High FoxP3 expression in tumour cells predicts better survival in gastric cancer and its role in tumour microenvironment. Br J Cancer 110: 1552-1560, 2014.

7. Ma GF, Chen SY, Sun ZR, Miao Q, Liu YM, Zeng XQ, Luo TC, Ma LL, Lian JJ and Song DL: FoxP3 inhibits proliferation and induces apoptosis of gastric cancer cells by activating the apoptotic signaling pathway. Biochem Biophys Res Commun 430: 804-809, 2013.

8. Hao Q, Zhang C, Gao Y, Wang S, Li J, Li M, Xue X, Li W, Zhang $\mathrm{W}$ and Zhang Y: FOXP3 inhibits NF- $\mathrm{B}$ activity and hence COX2 expression in gastric cancer cells. Cell Signal 26: 564-569, 2014

9. Chen Q, Zhang A, Yu F, Gao J, Liu Y, Yu C, Zhou H and Xu C: Label-free proteomics uncovers energy metabolism and focal adhesion regulations responsive for endometrium receptivity. J Proteome Res 14: 1831-1842, 2015.

10. Soderblom EJ, Philipp M, Thompson JW, Caron MG and Moseley MA: Quantitative label-free phosphoproteomics strategy for multifaceted experimental designs. Anal Chem 83: 3758-3764, 2011.

11. Wiśniewski JR, Nagaraj N, Zougman A, Gnad F and Mann M: Brain phosphoproteome obtained by a FASP-based method reveals plasma membrane protein topology. J Proteome Res 9: 3280-3289, 2010.

12. Livak KJ and Schmittgen TD: Analysis of relative gene expression data using real-time quantitative PCR and the 2(-Delta Delta C(T)) method. Methods 25: 402-408, 2001.

13. Liu C, Li Y, Semenov M, Han C, Baeg GH, Tan Y, Zhang Z, Lin $\mathrm{X}$ and He X: Control of beta-catenin phosphorylation/degradation by a dual-kinase mechanism. Cell 108: 837-847, 2002.

14. Gonzalez DM and Medici D: Signaling mechanisms of the epithelial-mesenchymal transition. Sci Signal 7: re8, 2014.

15. Kalluri R and Weinberg RA: The basics of epithelial-mesenchymal transition. J Clin Invest 119: 1420-1428, 2009.

16. Imhof BA, Vollmers HP, Goodman SL and Birchmeier W: Cell-cell interaction and polarity of epithelial cells: Specific perturbation using a monoclonal antibody. Cell 35: 667-675, 1983.

17. Polakis P: Wnt signaling and cancer. Genes Dev 14: 1837-1851, 2000.

18. Yamada S, Pokutta S, Drees F, Weis WI and Nelson WJ: Deconstructing the cadherin-catenin-actin complex. Cell 123: 889-901, 2005.

19. Nawshad A, Lagamba D, Polad A and Hay ED: Transforming growth factor-beta signaling during epithelial-mesenchymal transformation: Implications for embryogenesis and tumor metastasis. Cells Tissues Organs 179: 11-23, 2005.

20. Kemler R, Hierholzer A, Kanzler B, Kuppig S, Hansen K, Taketo MM, de Vries WN, Knowles BB and Solter D: Stabilization of beta-catenin in the mouse zygote leads to premature epithelial-mesenchymal transition in the epiblast. Development 131: 5817-5824, 2004.

21. MacDonald BT, Tamai K and He X: Wnt/beta-catenin signaling: Components, mechanisms, and diseases. Dev Cell 17: 9-26, 2009.

This work is licensed under a Creative Commons Attribution-NonCommercial-NoDerivatives 4.0 International (CC BY-NC-ND 4.0) License. 